\title{
Abstracts of Award-Winning Posters, 19th Annual Health Sciences Poster Conference, Faculty of Medicine, Health Sciences Centre, Kuwait University, Kuwait, May 6-8, 2014
}

\section{Dr. Nael Al-Naqeeb Undergraduate Research Award}

\section{1 \\ Public Knowledge and Attitudes Regarding Organ Donation in Kuwait \\ B. Bosakhar, D. Arab, N. Al-Ali, N. Al-Tawheid, S. Al-Farhan, Z. Al-Mesailekh, A.K. Mitra \\ Department of Community Medicine and Behavioural Sciences, Faculty of Medicine, Kuwait University, Kuwait}

Introduction: In Kuwait, the rate of organ donation is relatively low. The aims of this study included: (1) to evaluate public knowledge and attitudes on organ donation; and (2) to determine factors which predict public knowledge regarding organ donation. Methods: A cross-sectional study was conducted among 630 participants recruited from 27 randomly selected public cooperative societies and private supermarkets in Kuwait from December 2013 to January 2014. A self-administered questionnaire was used to collect data on demographics, public knowledge, attitudes, and their perceived barriers. The study was approved by the ethics committee, and an informed consent was taken from each participant before enrollment. Results: $68 \%$ knew about organ donation; however, more females than men knew about it ( 73 vs. $63 \%$, respectively, $\mathrm{p}=0.01)$. A composite score of knowledge was also higher among females than males $(8.4 \pm 5.8$ vs. $6.8 \pm 5.8$, respectively, $\mathrm{p}=0.001)$. People with a higher education had a better perception about organ donation ( $\mathrm{p}<0.001)$, and were more willing to donate an organ $(\mathrm{p}=0.04)$. Among the barriers, more females mentioned about health complications after organ donations (77 vs. $67 \%, p=0.011$ ) and a fear of the operation itself ( 83 vs. $69 \%$, $\mathrm{p}<0.001)$. In a multiple logistic regression analysis, female gender $(\mathrm{OR}=1.7 ; 95 \% \mathrm{CI}=1.2,2.4)$, and an educational level of bachelor's degree or higher $(\mathrm{OR}=2.6,95 \% \mathrm{CI}=1.7,3.9)$ were significant predictors of the knowledge about organ donation. Conclusions: In this study, females and people with bachelor's degree or a higher educational level were more knowledgeable about organ donation. Acceptance of organ donation was not correlated with socio-demographic factors; however, the willingness to donate organs was higher among people with higher educational level. Fear of the operation itself, health complications, and lack of proper knowledge were the most common perceived barriers of organ donation.

\section{2}

Low-Dose Exposure to Lead during Pregnancy Affects Spatial Learning and Neurogenesis in Hippocampus in Young Rats

H.M. Al-Shimalia, ${ }^{a}$, A.F. Al-Musaileem ${ }^{a, b}$, M.S. Rao ${ }^{b}$, K.M. Khan ${ }^{b}$

a 3 rd year Medical Students, ${ }^{b}$ Department of Anatomy, Faculty of Medicine, Kuwait University, Kuwait

Introduction: Lead $(\mathrm{Pb})$ is toxic to all organ systems. The severity of toxicity depends on the dose, duration of exposure, and developmental stage of the subject. Central nervous system has received much attention because of the impact of $\mathrm{Pb}$ exposure on cognitive and behavioral development in children. The aim of this study was to investigate the effects of in utero low-dose exposure to $\mathrm{Pb}$ on spatial learning and neurogenesis in hippocampus of young

\begin{tabular}{ll}
\hline KARGER & $\begin{array}{l}\text { (c) 2014 S. Karger AG, Basel } \\
\text { 1011-7571/14/0236-0587\$39.50/0 Openger }\end{array}$ \\
$\begin{array}{l}\text { E-Mail karger@karger.com } \\
\text { www.karger.com/mpp }\end{array}$ & $\begin{array}{l}\text { This is an Open Access article licensed under the terms of the } \\
\text { Creative Commons Attribution-NonCommercial 3.0 Un- } \\
\text { ported license (CC BY-NC) (www.karger.com/OA-license), } \\
\text { applicable to the online version of the article only. Distribu- } \\
\text { tion permitted for non-commercial purposes only. }\end{array}$
\end{tabular}


rats. Methods: Wistar rats were mated and after pregnancy was established, animals $(\mathrm{n}=10)$ were given drinking water with $0.1 \%$ lead acetate from day one of pregnancy until parturition. Control animals $(n=10)$ received deionized water. Spatial learning and memory of two pups from each dam were determined with Morris water maze on postnatal days 21 and 30. Animals were later sacrificed and $\mathrm{Pb}$ level in hippocampus was measured by atomic absorption spectrophotometer. Sections of hippocampus were stained for doublecortin (marker for neurogenesis). Data were analyzed with Student's t-test. Results: $\mathrm{Pb}$ in hippocampus of $\mathrm{Pb}$-exposed rats were significantly $(p<0.001)$ higher in 21 -day group but not in the 30 -day group. Water maze test revealed significant $(\mathrm{p}<0.01)$ memory deficit when tested on day 21 , but not when tested on day 30 . When retested for memory retention after 10 days both control and $\mathrm{Pb}$-exposed rats failed to locate the platform. However, after a session of relearning and retesting 24 hours later, only the rats from the control group located the platform. Pb-treated rats showed significantly decreased number of doublecortin positive neurons in the hippocampus in comparison to control rats $(\mathrm{p}<0.0001)$. Conclusions: Our data show that low-dose exposure to $\mathrm{Pb}$ during pregnancy results in impairment of spatial learning correlated with decreased neurogenesis in the hippocampus. Acknowledgements: We acknowledge the use of Research Core Facility (SRUL 02/13) and technical help of S. Jacob, S. Joe and S. Shivanandan.

\section{3 \\ Anti-Proliferative Activity of 5-Triazolylmethyl- and 5-Acetamido-Oxazolidinone Derivatives}

O.M. Hedaya, P.M. Mathew, F. Hassan, O.A. Phillips, Y.A. Luqmani

Department of Pharmaceutical Chemistry, Faculty of Pharmacy, Kuwait University, Kuwait

Introduction: In the face of increasing resistance to existing antibiotics, oxazolidinones, exemplified by linezolid, have been developed as promising agents. In this study we investigated several 5-triazolylmethyl- and 5-acetamido-morpholino and N-substituted-piperazino oxazolidinone derivatives for any antiproliferative activity against breast cancer and normal cells. Methods: MTT assay, validated by cell counting, was used to assess the effect of 12 derivatives (concentrations from 100nM to $10 \mathrm{microM}$ ) on the proliferation of MCF7 breast cancer cells. The three most active compounds were then tested on MDA231 breast cancer cells and HBL100 normal breast cells. Cytotoxicity of those selected was determined by assessing the extent of apoptosis by flowcytometry. The anti-metastatic potential of these compounds was assessed on MDA231 by the wound healing and agarose invasion assay. Results: The 5-triazolylmethyl piperazino-oxazolidinone derivatives containing 4-N-(2-chlorocinnamoyl), 4-N-(4-nitrobenzoyl) and $4-\mathrm{N}$-methylsulfonyl moieties showed the most potent cytostatic activity against the cancer and normal cells, inhibiting proliferation by up to $70 \%$, in the same order of their reported antibacterial activity against $\mathrm{S}$. aureus, but at much higher concentrations. Unexpectedly, there was a significant stimulation of proliferation at $100 \mathrm{nM}$, well below their antibacterial MIC. The compounds also retarded motility and invasion of MDA231 cells. Conclusions: Nine of the derivatives tested showed no effect on any of the eu- karyotic cell lines, which also shows their preferential activity against bacteria. Three compounds, however, exhibited potent cytostatic activity against both normal and cancer cells. Their biphasic response suggests multiple targets on eukaryotic cells. Used solely to treat bacterial infection they may encounter unwanted side effects. However, at $>10$ microM these may be further studied as anti-cancer agents because of their anti-metastatic properties.

\section{Best Postgraduate Awards}

\section{Graduate MSc (Basic Science)}

\section{1}

\section{Distribution of Virulence Genes in Helicobacter pylori Cultured from Native Arab Kuwaiti Patients with Dyspepsia}

H. Al-Abkal ${ }^{a, b}$, I. Siddique ${ }^{c}$, J. Al-Alic, T.A. Junaid d, M.J. Albert ${ }^{a}$

aDepartment of Microbiology, Faculty of Medicine, Kuwait University, Kuwait; ${ }^{\mathrm{b}}$ Arabian Gulf University, Bahrain;

Departments of ${ }^{\mathrm{C}}$ Medicine and ${ }^{\mathrm{d} P a t h o l o g y}$, Faculty of Medicine, Kuwait University, Kuwait

Introduction: $H$. pylori, the causative agent of several upper gastrointestinal diseases, possesses a number of virulence genes. The objective of the study is to determine the relationship between a set of virulence genes and the histopathology of gastric mucosa. We have completed the study of virulence genes of the isolates which is presented here. Methods: Three biopsies from the stomach ( 2 from antrum and 1 from corpus) were obtained from 141 consecutive, adult, native, Arab Kuwaiti patients with dyspepsia seen at Al-Amiri Hospital or Mubarak Al-Kabir Hospital, Kuwait, during Nov 2012-Feb 2013. Biopsies were cultured on selective agar microaerobically. Chromosomal DNA originating from a single colony was used as the template in PCR assay for the detection of virulence genes encoding the following factors: cag A and its Cterminal motif (EPIYA) subtypes, vacA and its various subtypes, OipA, and iceA and its alleles. Results: $H$. pylori was cultured from 50 out of 141 patients studied. The distribution of virulence genes in the $50 \mathrm{H}$. pylori isolates are as follows: cagA,36 (72\%) with EPIYA motif detected in 24 (48\%) with B type (15 isolates), A type (4 isolates), $\mathrm{A}$ and $\mathrm{B}$ type (3 isolates), $\mathrm{B}$ and $\mathrm{C}$ type (1 isolate) and $\mathrm{C}$ type (1 isolate); vacA 50(100\%) with its subtypes, vacAm1, 3 (6\%), vacAm2, 36 (72\%), vacAs1, 16 (32\%), vacAs2, 23 (46\%), vacAs1a, 29 (58\%), vacAs1b, 24 (48\%), vacAs2, 35 (70\%), vacAi1, 13 (26\%), vacAi2, 39 (78\%), vacAd1, 10 (20\%), and vacAd2, 33 (66\%); oipA, 41 (82\%); and iceA1, 10 (20\%) and iceA2, 13 (26\%). Conclusions: This study has demonstrated that $H$. pylori infecting the native, $\mathrm{Ku}$ waiti patients possess a number of virulence genes. In other studies, presence of some of these genes was correlated with the development of gastritis, gastric ulcer or gastric cancer. When the histopathologic study on the biopsies of these patients is complete, it will be possible to correlate the presence of these virulence factors with the disease outcome. Funding Agency: Enrolled for MSc at Arabian Gulf University, Bahrain, partly funded by the University. 


\section{Graduate Resident}

1

\section{Study of Patatin-Like Phospholipase-3/Adiponutrin I148M Polymorphism and Biochemical Markers in Nonalcoholic Fatty Liver Disease}

\begin{abstract}
A.K. AlAnjeri' ${ }^{a}$ A.E. Al Serrib ${ }^{b}$, R. Gupta ${ }^{c}$, H.A. El Baaly ${ }^{\text {, }}$, P. Kinatamitath ${ }^{e}$, O. Shaarawi ${ }^{f}$, O.A. Mojiminiyie

${ }^{a}$ Clinical Biochemistry Laboratory, Mubarak Hospital, bHuman Genetics Unit, Department of Pathology, Faculty of Medicine, Kuwait University, 'Department of Radiology, Faculty of Medicine, Kuwait University, ${ }^{\mathrm{d}}$ Radiology Department, Mubarak Hospital, e'Department of Pathology, Faculty of Medicine, Kuwait University, and ${ }^{\mathrm{f}}$ Apheresis Unit, Kuwait Central Blood Bank, Kuwait
\end{abstract}

Introduction: Nonalcoholic fatty liver disease (NAFLD) is the most common liver disease. Some of gene polymorphisms have been reported to be associated with NAFLD. We hypothesize that the single-nucleotide polymorphism rs738409 C>G at position 148 in the patatin-like phospholipase-3 gene (PNPLA3) will increase the susceptibility of NAFLD. This study aims to examine the association of PNPLA3 polymorphism with the prevalence and severity of NAFLD among Kuwaiti lipid clinic patients and to study the association of NAFLD with markers of liver damage including alanine aminotransferase, alkaline phosphatase, gamma glutamyl transferase, total bilirubin, and tissue inhibitor of metalloproteinases-1. Methods: 78 Kuwaiti lipid clinic patients were enrolled. Their anthropometric parameters, chemistry profile, TIMP-1, PNPLA3 gene and abdominal ultrasound were analyzed. Statistical analyses were performed with SPSS 20.0. Mean values were compared by analysis of variance. Binary logistic regression analysis was used to ascertain the association of variables with the NAFLD and metabolic syndrome. A P-value $<0.05$ was considered as statistically significant. Results: The I148M variant of PNPLA3 gene and markers of liver damage were not associated with NAFLD. Moderate-to-severe hepatic steatosis was significantly associated with low high-density lipoprotein-cholesterol ( $p$ $=0.007)$, high alanine aminotransferase $(\mathrm{p}=0.034)$ and $\mathrm{G}$ allele $(p=0.009)$. Patients carrying the CG and GG genotypes had significantly higher levels of total bilirubin $(\mathrm{p}=0.011)$ and more severe degrees of hepatic steatosis $(p=0.042)$ compared to those carrying the CC genotype. Conclusions: The I148M variant of PNPLA3 gene and biochemical markers of liver damage are not associated with NAFLD in Kuwaiti lipid clinic patients. The mutant $\mathrm{G}$ allele is a risk factor for severe grades of hepatic steatosis in patients with NAFLD.

\section{Atrial Myxoma Presenting with Symptoms of Palpitation in a 55 Year-Old Caucasian Male: A Case Report}

T. Essam Eldin, K. Mahmoud, B. Al Enezi

Al Jahra Hospital, Kuwait

Background: Atrial myxomas are the most common primary cardiac tumors. They are usually small or moderate in size by the time of the diagnosis, exhibiting nonspecific cardiac or systemic symptoms, and are most frequently soft and friable without microscopic signs of ossification. We describe herein rare case of a large left atrial myxoma with hemorrhage inside the tumor. Case Summary: A 58-year-old male with history of hypertension, was referred to the cardiology clinic because of palpation of 2 months duration. An ECG was done which showed normal sinus rhythm, left ventricular hypertrophy and infrequent PVCs. An echo-cardiography was done to rule out hypertensive heart disease. A $4 \times$ $4 \mathrm{~cm}$ mobile mass was seen in the left atrium attached to the interatrial septum and protruding into the left ventricle. The patient was referred to the cardiac surgeon, and a coronary angiography was done prior to the surgery revealing normal coronaries. The mass was excised and pathological examination revealed a benign atrial tumor (myxoma) measuring $5 \times 4 \mathrm{~cm}$ with hemorrhage within the mass and a gelatinous capsule at the top of the mass. Conclusion: We consider our case as extremely rare because of the asymptomatic course despite the large size of the tumor, with obstruction of the mitral orifice.

\section{Graduate PhD (Basic Science)}

\section{1}

\section{Characterization of Methicillin-Resistant Staphylococcus Aureus in Kuwait Hospitals: 1992-2010}

\section{S.S. Boswihi, E.E. Udo, N. Al-Sweih}

Department of Microbiology, Faculty of Medicine, Kuwait University, Kuwait

Introduction: Methicillin-resistant Staphylococcus aureus (MRSA) have been isolated in Kuwait hospitals since 1992. However, there have been no comprehensive studies of their genotypes. The aim of this study was to investigate the distribution of MRSA clones obtained from patients in Kuwait hospitals from 1992 to 2010. Methods: Four hundred MRSA isolates representing prevailing PFGE types obtained from 13 hospitals in Kuwait were characterized using SCCmec typing, spa typing and multi locus sequence typing. Results: In total, 57 spa types and 27 sequence types were detected among isolates studied. The dominant genotypes were ST239-III-t421 (86; 21.5\%), ST241-III-t037 (43; 10.7\%), ST239-III-t945 (28; 7.0\%), ST239-III-t037 (26; 6.5\%), ST239-IIIt860 (18; 4.5\%), ST22-IV-t223/t852/t032 (33.0;8.2\%), ST80-IVt044/t045/t376 (27;6.7\%), ST30-IV-t019/t318/t345 (14;3.5\%), ST5-II-t003/t688 (20; 5.0\%), ST6-IV-t304 (9;2.5\%) and ST36-IV- 
t018/t605 (8; 2.0\%). Other genotypes occurred less frequently. The number of MRSA genotypes increased from one (ST239-III-t037) in 1992 to four in 1996, eight in 1999, six in 2001, 11 in 2005 and 23 in 2010. While the ST239-III-t037 genotype was detected in 1992, 1996 and 2005, the ST239-III-t421 was detected from 1992 to 2010. ST241-III-t037 genotype was detected from 1997 to 2002. The ST30-IV-t019 was first detected in 1996 while ST80-IV-t044 first appeared in 1997. Among the ST22 isolates, the ST22-IV-t032 was detected in 2005, while ST22-IV-t223 was detected in 2005 and 2010. Novel genotypes detected in 2010 included ST772-V-t657, ST8-IV-t008, and ST88-IV-t690. Conclusions: The study revealed changes in the numbers and diversity of MRSA clones in Kuwait hospitals over time. Although a diversity of new MRSA clones were observed in 2005 and 2010, the ST239-III-t421 genotype remained the dominant MRSA clone in Kuwait hospitals. Funding Agency: Research Administration, grant no. YM 02/12.

\section{Basic Sciences}

\section{1 \\ Characterization of Gallbladder Stone, Bile and Tissue Using Time Resolved Fluorescence Spectroscopy}

\author{
A. Shuaib ${ }^{a}$, J. Kokajb , G. Pichler ${ }^{b}$, R. Nair ${ }^{b}$, M. Joseph ${ }^{b}$

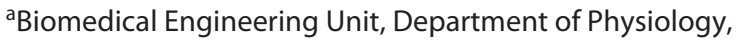 \\ Faculty of Medicine, Kuwait University, and ${ }^{\text {b}}$ Department of \\ Physics, Faculty of Science, Kuwait University, Kuwait
}

Introduction: Laser-induced lithotripsy of gallbladder stone has the potential in expanding significantly with laser system, endoscopes, retrieval devices and modern imaging devices. The laser efficiency is the key parameter, such as pulse power, pulse width, and number of pulses, which are specific for different types of stones. In addition, a guiding tool will be used to assist in discriminating the stone, the bile and the surrounding tissue. Time Resolved Fluorescence Spectroscopy (TRFS) can accommodate all these parameters and could be a new milestone in laser based lithotripsy. Methods: A femtosecond laser (wavelength $=400 \mathrm{~nm}$ ) was used in this study. A bifurcated fiber was used for excitation as well as for fluorescence signal collection. The collected laser induced fluorescence light was then dispersed by a spectrometer, which is coupled to the streak camera detection system. Time resolved spectral data of the fluorescence signal was recorded to the computer for further analysis. For wavelength-domain comparison of the stone fluorescence signal, we used Ocean optics spectrometer. We also used inductively coupled plasma system to identity the stone chemical composition quantitatively. Results: Spectral- and time-resolved features were used to develop a new classification algorithm. The algorithm can be used to characterize gallbladder stones, and discriminate stone, bile and tissue. Moreover, the algorithm can be extended to set the most appropriate parameters for the laser lithotripsy, which is needed by the physicians in real-time stone fragmentation. Conclusions: This study demonstrates TRFS capable of characterizing and optimizing parameters in laser based lithotripsy. It can be one of the essential guiding tools in modern laser lithotripsy. Further research should be carried out to develop a single probe system to identify the type of stone and automatically set the optimum laser parameters in real-time laser lithotripsy of gallbladder stones.

\section{Clinical}

\author{
1 \\ Efficacy of Botulinum Toxin-A Treatment in Chronic \\ Migraine - First Middle East Experience \\ J.Y. Al-Hashella,b V. Nagarajan ${ }^{a}$, S.F. Ahmed ${ }^{a, c}$ \\ a Department of Neurology, Ibn Sina Hospital, Kuwait, \\ ${ }^{b}$ Department of Medicine, Faculty of Medicine, Kuwait \\ University, Kuwait; ' Department of Neurology and Psychiatry, \\ Faculty of Medicine, Minia University, Minya, Egypt
}

Introduction: BoNT-A is approved for prophylactic treatment of CM. We aimed to assess the efficacy and safety of Botulinum toxin-A (BoNT-A) in the treatment of chronic migraine (CM). Methods: This open-label prospective study included $40 \mathrm{CM}$ patients. Each patient received 100 units of BoNT-A following fixed site fixed dose protocol. Patients' headache was assessed by their headache diary and recording Headache Impact test (HIT-6) at baseline and 4, 8 and 12th weeks following BoNT-A injection. Adverse events (AEs) were monitored to assess the efficacy of BoNTA. For willing patients, BoNT-A injection was given and they were assessed at 3 months' interval. Results: After BoNT-A treatment, there were reductions in all parameters (headache frequency and severity, analgesic consumption and HIT-6 score) by $35-40 \%$ at 4 th week, $41-45 \%$ at 8 th week and $39-42 \%$ at 12 th week post treatment. At 4 th week, $62.5 \%$ of patients achieved good response while $37.5 \%$ indicated no alteration in their headache frequency and severity. At 8 th week and 12 th week post treatment $30,25 \%$ respectively were found to have no response to treatment. Five patients (12.5\%) experienced mild and short lasting AEs. There was 60$70 \%$ improvement of variables after repeated injections at 3 months' interval. Conclusions: BoNT-A is effective and well tolerated therapy in the prophylaxis of CM. 